Decision Support

\title{
A comprehensive method for comparing mental models of dynamic systems
}

\author{
Martin Schaffernicht ${ }^{\mathrm{a}}$, Stefan N. Groesser ${ }^{\mathrm{b}, *}$ \\ ${ }^{a}$ Universidad de Talca, Facultad de Ciencias Empresariales, Avenida Lircay s/n, 3460000 Talca, Chile \\ ${ }^{\mathrm{b}}$ University of St. Gallen, Institute of Management, System Dynamics Group, Dufourstrasse 40a, 9000 St. Gallen, Switzerland
}

\section{A R T I C L E I N F O}

\section{Article history:}

Received 15 March 2010

Accepted 1 September 2010

Available online 8 September 2010

\section{Keywords:}

Problem structuring

Mental models

Dynamic systems

Feedback

\begin{abstract}
A B S T R A C T
Mental models are the basis on which managers make decisions even though external decision support systems may provide help. Research has demonstrated that more comprehensive and dynamic mental models seem to be at the foundation for improved policies and decisions. Eliciting and comparing such models can systematically explicate key variables and their main underlying structures. In addition, superior dynamic mental models can be identified. This paper reviews existing studies which measure and compare mental models. It shows that the methods used to compare such models lack to account for relevant aspects of dynamic systems, such as, time delays in causal links, feedback structures, and the polarities of feedback loops. Mental models without those properties are mostly static models. To overcome these limitations of the methods to compare mental models, we enhance the widely used distance ratio approach (Markóczy and Goldberg, 1995) so as to comprehend these dynamic characteristics and detect differences among mental models at three levels: the level of elements, the level of individual feedback loops, and the level of the complete model. Our contribution lies in a new method to compare explicated mental models, not to elicit such models. An application of the method shows that this previously non-existent information is essential for understanding differences between managers' mental models of dynamic systems. Thereby, a further path is created to critically analyze and elaborate the models managers use in real world decision making. We discuss the benefits and limitations of our approach for research about mental models and decision making and conclude by identifying directions for further research for operational researchers.
\end{abstract}

(c) 2010 Elsevier B.V. All rights reserved.

\section{Introduction}

This paper provides means for comparing mental models of dynamic systems. Generally speaking, a mental model is an abstract representation of a situation or a system in the mind of an individual (Forrester, 1961, 1992). "Mental models reflect the beliefs, values, and assumptions that we personally hold, and they underlie our reasons for doing things the way we do" (Maani and Cavana, 2007: 15). They are used for inductive and deductive reasoning (Johnson-Laird, 1999, 2001). Doyle and Ford $(1998,1999)$ have defined a mental model of a dynamic system (MMDS) as "a relatively enduring and accessible, but limited, internal conceptual representation of an external system (historical, existing, or projected) whose structure is analogous to the perceived structure of that system" (Doyle and Ford, 1999: 141). ${ }^{1}$

Mental model research is relevant in operational research (Eden, 1988; Howick et al., 2008), management (Lim and Klein, 2006; Markóczy and Goldberg, 1995; Tyler and Gnyawali, 2009), and organizational research (Calori et al., 1994; Fokkinga et al., 2009; Rouwette et al., 2002; Smith-Jentsch et al., 2001). This is especially the case where enhancing the accuracy of decision makers' mental models of either a situation or a system is one of the goals of modelling. There is evidence that the accuracy of mental models indeed moderates performance when acting in such systems (Capelo and Dias, 2009; Gary and Wood, forthcoming; Jonson and O'Connor, 2008). However, when feedback-driven dynamic systems come into play, research lacks a comprehensive method to compare mental models which include the essential conceptual elements of such systems. Conceptual elements are categorical building blocks used to formalise the content of a mental model.

Up to now, comparative mental model research has used several approaches to account for the models' conceptual structure: the distance ratio approach (Markóczy and Goldberg, 1995), the closeness ratio approach (Schvaneveldt, 1990), or related approaches (Doyle et al.,

\footnotetext{
* Corresponding author. Tel.: +41 71224 2379; fax: +41 712242355.

E-mail addresses: martin@utalca.cl (M. Schaffernicht), stefan.groesser@unisg.ch (S.N. Groesser).

${ }^{1}$ In this paper, we use 'mental models' or 'models' to refer to MMDS.
} 
2008; Gary, 2008; Gary and Wood, forthcoming). Even though these approaches have yielded significant insights about differences in mental models, they do not account for essential conceptual elements of dynamic systems, especially delayed causal effects, feedback loops, and the polarity of these feedback loops. These three elements complement the definition of a MMDS developed by Doyle and Ford (1998). Currently, additional research is ongoing which explicates all components required to represent feedback-driven dynamic systems (Groesser and Schaffernicht, 2010). It is this paper's purpose to develop a comprehensive method for comparing MMDS incorporating all of the aforementioned elements of dynamic systems. There are significant benefits of such a comparison method: mental models can be analyzed more comprehensively and differences between mental models can be detected at three levels: the level of the elements (element distance ratio, EDR), the level of the individual feedback loop (loop distance ratio, $L D R$ ), and the level of the complete model (model distance ratio, $M D R$ ). Furthermore, the method proposed here can be used to compare several versions of mental models both within and between subjects. We perceive the method as a stepping stone for rigorous and cumulative research about mental model comparison.

This article is structured in the following way: In Section 2, we review existing literature on measurement and comparison of MMDS. Section 3 develops our method to compare mental models, which is then applied in Section 4. For this application, we use mental models which have been already elicited and are represented by feedback loop diagrams. The discussion in Section 5 focuses on how the new method adds to the existing research, how it improves the capability for interpreting the differences between MMDS, and how the current version can be extended. Section 6 concludes the paper and provides paths for future research.

\section{Literature review}

Research about mental models has addressed such issues as elicitation, measurement, and comparison of mental models as well as the improvement of their accuracy. We have reviewed existing studies about measuring and comparing mental models to find out what conceptual elements of a mental model have been taken into account. Studies have assessed the degree of similarity between either models of different subjects, known as the between-subjects approach, or between different versions of a model of one subject before and after an intervention, known as the within-subject approach. Most studies that measure and compare changes of the accuracy of models employ the within-subject approach; only a few apply the between-subject approach. First, we have reviewed the former type of studies, which also includes measuring models and comparing them to an ideal, computational model of reality. Then, we review the latter type of studies (Table 1). Here, it is not required to report the literature about elicitation of mental models or about mental model improvements (for instance, Carley and Palmquist, 1992).

We have started out with a review of intervention studies which have used the methodology of group model building (Andersen et al., 1997; Richardson and Andersen, 1995; Vennix, 1996). Rouwette et al. (2002) conducted a meta-analysis of 107 studies published between 1981 and 1999, which shows that outcomes of interventions are assessed in different ways. A variety of methods such as observations, interviews, and questionnaires have been used to assess the impact of group model building interventions on such concepts as shared language, commitment, and behavioural changes. This approach yielded a large number of mainly incomparable case descriptions. According to Rouwette et al. (2002), only Verburgh's (1994) study, one out of these 107, explicitly addressed the effect of group model building interventions on participants' mental models. For his assessment, he measured the number of concepts, relationships, and feedback loops of the individuals' mental models. In addition, he accounted for the change in time indications, the lengths of the causal paths, and the density of the mental model. Until 2009, most of the intervention studies did not attempt to measure the effects of interventions on participants' MMDS (e.g., Bosch et al., 2003; Snabe and Grössler, 2006; Weber and Schwaninger, 2002; Woog et al., 2006). Often these studies argue only qualitatively for the benefits of interventions and the effects on the participants' MMDS. An exception is a study by Fokkinga et al. (2009). For the measurement of the changes in the mental models of 44 individuals, they have used an intervention study design. Their comparison has included the number of variables in the individual models, which are also part of the group mental model, and the number of feedback loops. The results suggest that the number of variables has increased significantly due to the intervention; no change has been detected in the number of shared variables or the number of feedback loops, however.

Our review of the relevant literature also unearthed studies which follow intervention approaches other than group model building. Doyle et al. (1996) have compared mental models of 25 undergraduates to find out if the intervention by means of a simulation game about the economic long wave (Sterman, 1985) altered the mental models used by the students to reason about the economic cycle. Based on a rigorous design for intervention and measurement, the comparison of the coded mental models accounts for the number of variables, the

Table 1

Studies about the comparison of mental models of dynamic systems.

\begin{tabular}{|c|c|c|c|}
\hline Authors & Study design & Elements accounted for & Approach \\
\hline Plate (2010) & Between-Subject & Link density, web-like causality index & Own approach \\
\hline Fokkinga et al. (2009) & $\begin{array}{l}\text { Within- and Between- } \\
\text { Subject }\end{array}$ & Variables, variables similar to group model, feedback loops & Own approach \\
\hline Doyle et al. (1996) & Within-Subject & Variables, causal links, feedback loops, polarities & Own approach \\
\hline Doyle et al. (2008) & Within-Subject & $\begin{array}{l}\text { Variables, causal links, feedback loops, polarities, } \\
\text { normalized maximal path length, normalized length } \\
\text { of feedback loop }\end{array}$ & Own approach \\
\hline Verburgh (1994) & Within-Subject & $\begin{array}{l}\text { Variables, causal links, feedback loops, path length, } \\
\text { density of the MM, time indications }\end{array}$ & Own approach \\
\hline Gary (2008) and Gary and Wood (forthcoming) & Between-Subject & Causal links, polarities, behaviour of systems, feedback loops & Closeness ratio \\
\hline Capelo and Dias (2009) & Between-Subject & Common links & Closeness ratio \\
\hline Ritchie-Dunham (2002) & Between-Subject & Common links & Closeness ratio \\
\hline Langfield-Smith and Wirth (1992) & Between-Subject & Variables, causal links, polarities & Distance ratio \\
\hline Markóczy and Goldberg (1995) & Between-Subject & Variables, causal links, polarities & Distance ratio \\
\hline Langan-Fox et al. (2000) and Langan-Fox et al. (2001) & Between-Subject & Variables, causal links, polarities & Distance ratio \\
\hline Langley and Morecroft (2004) & No comparison & not reported & Own approach \\
\hline
\end{tabular}


number of connections between variables, and the amount of feedback relationships. Building upon this study, Doyle et al. (2008) have further elaborated their measurement instrument. As before, they have used a computer simulation as intervention means, and the mental models from 46 subjects were analyzed regarding the number of events, the number of links, the number of links per event, and also the average length of the causal paths extending back from the primary to-be-explained events. In addition, they have analyzed the empirical material for dynamic components of a mental model which were operationalized by the number of feedback loops. They divided the number of feedback loops by the average loop length to control for the fact that longer feedback loops can more likely be parts of additional feedback loops. They also accounted for remoteness in time and space by calculating the maximum causal path length extending back from the primary to-be-explained events divided by the maximum possible path length.

Others have investigated the similarity of MMDS without an intervention. The studies by Capelo and Dias (2009) and Plate (2010) compared the MMDS of the participants with an ideal model of a computational business system or expert models, respectively. For comparison, they have used the number of common links in both models, a method known as closeness approach (Ritchie-Dunham, 2002; Rowe and Cooke, 1995; Schvaneveldt, 1990).

Gary (2008), Gary and Wood (forthcoming), McGaghie et al. (2000), and Ritchie-Dunham (2002) have tested the effect of additional information about the feedback structure of a complex system on decision quality and found that mental model accuracy enhances performance when supported by feedback information. They have also measured the common links between pairs of variables, as perceived by the decision makers, and have compared them to the ideal computational model. Ritchie-Dunham (2002) subjects were presented with a list of related variables and were asked for their degree of relatedness. The degree of mental model similarity then was figured as the percentage of items on the knowledge test answered correctly. Gary (2008) and Gary and Wood (forthcoming) have extended this measurement, using two sets of knowledge questions that test for bivariate causal relationships, the polarity of these relationships, the number of variables in the mental model, the number of immediate feedback loops between two variables, and the behaviour that is generated by the model structure.

Studies by Langfield-Smith and Wirth (1992), Langan-Fox et al. (2000, 2001) and Markóczy and Goldberg (1995) employ the distance ratio approach to calculate the similarity of different individuals' mental models. They have computed the distances between the mental models taking into account the number of variables, the links between the variables as well as their polarities.

While these studies explicitly addressed the degree of similarity among mental models to reality, other investigations strove to understand the relationship between the accuracy of mental models relative to reality and the performance of the decision maker over time (e.g., Bakken et al., 1992; Bakken, 1993; Graham et al., 1992; Kluge, 2008; Kunc and Morecroft, 2007; Langley and Morecroft, 2004; Morecroft, 1992; Moxnes, 1998, 2004; Romme, 2004). These studies do not report the instrument to measure model accuracy or resulting model changes. Therefore, it is not fruitful to detail these studies here. In addition, we exclude studies that measure the process of thinking rather than measure MMDS. Such studies have been reviewed by Rouwette et al. (2004).

To summarize, research about MMDS has employed different approaches to measure and compare mental models. Most researchers have used existing instruments (Capelo and Dias, 2009; Markóczy and Goldberg, 1995; Ritchie-Dunham, 2002); they account for the number of variables, the number of common links, and the polarities of links. Others have developed their own instruments to measure the conceptual elements that they perceive a mental model contains (Doyle et al., 1996, 2008; Fokkinga et al., 2009; Gary, 2008; Gary and Wood, forthcoming).

As far as feedback-driven dynamic systems are concerned, we confirm that Forrester did not make explicit what a mental model contains (Forrester, 1961, 1968b), and that research on MMDS has mostly failed to account for the relevant characteristics of such systems. We maintain that time delays, feedback loops, and the polarity of loops must be part of the conceptual structure of mental models. It has been shown that not taking account of these characteristics as shown in Fig. 1 can lead to flawed understanding of system behaviour (Moxnes, 2004; Sterman, 2008; Sweeney and Sterman, 2007). As for the structure and differences of mental models from a feedback perspective, not much can be analyzed without a comparison method that accounts for the relevant characteristics, as has been outlined before. And what is more, two mental models may appear to be distinct when evaluated by a comparison of their variables and links, but are almost identical when compared at the level of feedback loops (Schaffernicht and Groesser, 2009). This underlines our argument that in a field that understands systems as being composed of structures that can be differentiated into three levels of description, models of the systems should be compared at all three levels. Fig. 1 shows this structure with its three levels: the model, the individual feedback loop and the elements; the mathematical ratios we use in this paper to denote the different levels are provided next to their respective levels.

\section{A method for comparing mental models of dynamic systems}

In this section, we introduce a method to compare MMDS. We describe the distance approach (Markóczy and Goldberg, 1995), which we have used as point of departure. Thereafter, we enhance the distance approach by accounting for delays between cause and effects as well as feedback loops and their polarities.

\subsection{Point of departure: The original distance ratio method}

The distance ratio approach is a beneficial point of departure for developing a comprehensive method to compare mental models (Schaffernicht et al. 2009). The approach was developed by Langfield-Smith and Wirth (1992) and Markóczy and Goldberg (1995). ${ }^{2}$ The comparison is based on the variables and links of two models. It divides the number of actual differences between both by the number of potential differences and produces a distance ratio that is a non-negative number that expresses the degree of difference between both models. The ratio ranges from [0] ("all variables and links are identical; thus, the models are identical") to [1] ("all variables and links are different, thus, the models have no commonalities"). The distance ratio approach has been used for system dynamics studies (Doyle et al., 1996, 2008) as well as in other research concerning managers' beliefs (Hine et al., 2005; Markoczy, 1997, 2000).

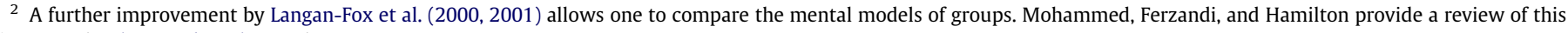
literature (Mohammed et al., 2010).
} 


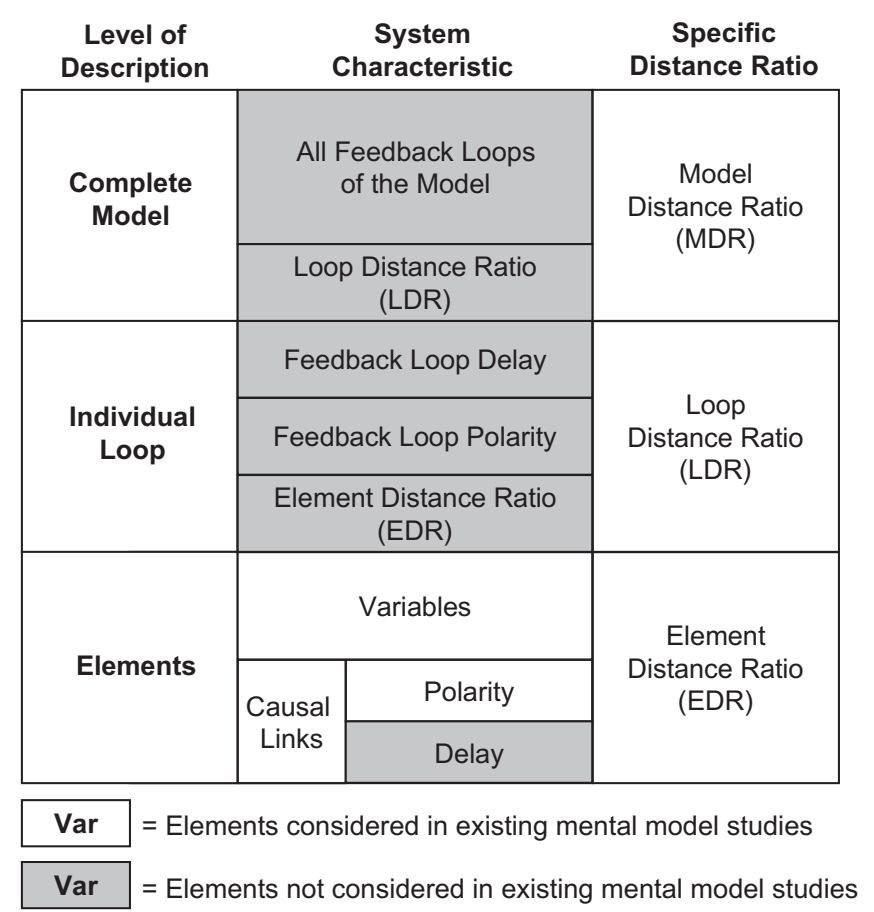

Fig. 1. Three levels of description and their respective distance ratios.

Let us suppose that there are two mental models $A$ and $B$ which have been elicited by a professional elicitation process and which are now to be compared. For our purposes, we conceive the elicitation process as professional when the mental models have been elicited and measured accurately without any introduction of errors or biases.

Each of the models consists of a number of variables, $v$, and a number of causal links with a polarity and a link strength. In what follows, each model is represented as an adjacency matrix $A$ and $B(v \times v)$ which contains all variables of both models. The indicator $V_{c}$ is the set of common variables in $A$ and $B$; $v_{c}$ is the amount of common variables; $v_{u A}$ is the amount of variables unique in $A$, and $v_{u B}$ the amount of variables unique in $B$, respectively.

The rows of $A$ and $B$ are numbered from 1 to $v$ using an index $i$, and columns from 1 to $v$ using index $j$. Each variable is assigned to a row and a column. Links between variables will appear in the elements of $A$ and $B$; positive integers denote a positive link polarity, while negative integers denote a negative link polarity. For instance, if in $A$ a link between the variables $r$ and $c$ exists, then cell $a_{r, c} \neq[0]$; if there is no causal relation between both variables, then $a_{r, c}=[0]$.

A number of parameters allow one to choose between several options for counting differences. Parameter $\alpha$ expresses the possibility of including self-loops in the model ([0] = possible, $[1]=$ not possible). A self-loop is given when a variable influences itself directly. Parameter $\beta$ represents the highest possible link strength, which accommodates the method to mapping approaches that elicit links with different strengths. Parameter $\gamma$ determines how differences between the sets of causal links in the two models are interpreted. Analysts may consider that nothing can be gained from the information that a link exists only in one of both models. However, if such differences are perceived to be meaningful, there are two possibilities: first, an individual may believe there is no link between two variables, or second, that one or both of the involved variables are not part of the mental model. For instance, if $A$ includes a link from variable $r$ to $c$, which does not exist in $B$, this may be because the individual believes that there is no causal relation (first case), or it might be that either $r$ or $c$ is not part of $B$ (second case). Parameter $\delta$ indicates, if a difference in polarity between two causal links is more important when the involved links have different strengths. Parameter $\varepsilon$ represents the number of possible polarities. The original distance ratio of Markóczy and Goldberg (1995) is provided in Eq. (1).

Equation 1: Original distance ratio formula (Markóczy and Goldberg, 1995).

$$
\begin{aligned}
& D R(A, B)=\frac{\sum_{i=1}^{v} \sum_{j=1}^{v} \operatorname{diff}(i, j)}{(\varepsilon \varepsilon+\delta) v_{c}^{2}+\gamma \prime\left(2 v_{c}\left(v_{u A}+v_{u B}\right)+v_{u A}^{2}+v_{u B}^{2}\right)-\alpha\left((\varepsilon \beta+\delta) p_{c}+\gamma^{\prime}\left(v_{u A}+v_{u B}\right)\right)}, \\
& \operatorname{diff}(i, j)= \begin{cases}0 & (i) \text { if } i=j \text { and } \alpha=1 ; \\
\Gamma\left(a_{i j}, b_{i j}\right) & \text { (ii) if either } i \text { or } j \notin V_{c} \text { and } i, j \in V_{A} \text { or } i, j \in V_{B} ; \\
\left|a_{i j}-b_{i j}\right|+\delta & (i i i) \text { if } a_{i j} b_{i j}<0 ; \\
\left|a_{i j}-b_{i j}\right| & (i v) \text { otherwise, }\end{cases} \\
& \Gamma\left(a_{i j}, b_{i j}\right)= \begin{cases}0 & (\text { a }) \text { if } \gamma=0 ; \\
0 & \text { (b) if } \gamma=1 \text { and } a_{i j}=b_{i j}=0 ; \\
1 & \text { (c) otherwise. }\end{cases}
\end{aligned}
$$


In Eq. (1), the numerator represents the number of relevant differences between the elements of $A$ and $B$. The denominator constitutes the largest possible number of differences that can exist between $A$ and $B$. The number of possible polarities, $\varepsilon$, and the possible importance of differences in polarities, $\delta$, multiply the common variables $v_{c}$. Then, two terms in the denominator contain information about $v_{u A}$ and $v_{u B}$; each depends on a parameter $\gamma^{\prime}$. If the fact that some variables belong to only one of the models is perceived to be meaningful, then $\gamma>[0]$ and $\gamma^{\prime}=[1]$. The respective terms will then express this difference; otherwise $\gamma^{\prime}=[0]$. In the following, we use the original distance ratio and enhance it to account for three additional components of dynamic systems.

\subsection{Incorporating delays between cause and effect at the element level}

Compared to a normal, non-delayed causal effect, a delayed causal effect approaches its normal link strength over a specified time, during which the strength of the link changes. The failure to perceive such delays undermines the individual's ability to notice and consider the nonlinear behaviour of a causal relation. To account for such delays, we re-interpret the parameter $\beta$ in such a way that it also expresses the fact that delayed links are different from normal links. This addition does not change Eq. (1) formally, but in terms of interpretative scope.

\subsection{Specification for system dynamics: The element distance ratio}

The study of mental models of dynamic systems using the system dynamics approach requires several specifications of the parameters, which have been introduced earlier. First, in system dynamics self-loops are not feasible (Richardson and Pugh, 1981); thus, $\alpha=[1]$. Parameter $\beta$ would usually be set to [1] since the assignment of different links strengths is not part of qualitative system dynamics (Sterman, 2000). Here our additional interpretation of $\beta$, as has been outlined just before, comes into play: we change $\beta$ from [1] to [2]; [1] represents a non-delayed link, and [2] a delayed link. These numbers do not indicate that a delayed link counts twice; rather, they express the fact that a delayed link and a non-delayed link are different. With regards to the interpretations of differences between the sets of causal links in both models, we believe that these differences are meaningful (as elaborated earlier); hence, $\gamma=[2]$. In system dynamics, the difference between the polarities of two identical links does not depend on other attributes, e.g., their strength; therefore, $\delta=[0]$. Moreover, only positive and negative polarities are possible, so $\varepsilon=[2]$. With this parameter configuration $(\alpha=[1], \beta=[2], \gamma=[2], \delta=[0], \varepsilon=[2])$, we obtain the distance ratio specified for the application of system dynamics, which we name: Element Distance Ratio, EDR. It expresses the degree of difference between two models at the level of variables and links including polarity and link delays (Eq. (2)).

Equation 2: The element distance ratio, EDR, as specified for system dynamics.

$$
\operatorname{EDR}(A, B)=\frac{\sum_{i=1}^{p} \sum_{j=1}^{p} \operatorname{diff}(i, j)}{4 v_{c}^{2}+\left(2 v_{c}\left(v_{u A}+v_{u B}\right)+v_{u A}^{2}+v_{u B}^{2}\right)-\left(2 v_{c}+\left(v_{u A}+v_{u B}\right)\right)}
$$

\subsection{Accounting for the level of feedback loops: The loop distance ratio}

Complex dynamic systems are driven by interacting feedback loops (Forrester, 1968b). A feedback loop is a logically closed causal chain where an initial change in a variable is fed back to its origin. When several such loops interact, some may be dominant at certain times, but then dominance might shift to other loops. These shifts in feedback loop dominance are the fundamental mechanisms that create the dynamic behaviour of a system (Richardson, 1999). In explicit models of such systems, feedback loops are represented at a higher level of description. Research demonstrates that a positive correlation exists between the number of feedback loops of an individual's mental model and the degree of dynamic thinking of that individual (Doyle et al., 2008; Senge, 1990; Sterman, 2008; Sweeney and Sterman, 2007). Therefore, a method to compare MMDS requires one to address the degree of distance between models at the level of feedback loops.

Before being able to calculate the distance of two models at the level of feedback loops, we must first define the loop sets of the models, and second, select the corresponding loops in both models. This is what we elaborate in the following. Just as the comparison of variables between two models requires two sets of variables, the comparison of feedback loops likewise requires two sets of feedback loops - one loop set for each model. Every MMDS has a non-negative amount of feedback loops; in small models, there might be only one loop. However, the number of loops increases exponentially as the amount of variables increases (Kampmann, 1996). Thereby, two or more simple and short feedback loops combine into larger, more complex loops (Oliva, 2004), building a hierarchy of more complex loops. Oliva offers a method for calculating the shortest independent loop set (SILS) by means of a graph-theoretical analysis (Oliva, 2004). That set is the smallest amount of the shortest loops which contains all variables which are elements of feedback loops in the mental model. One might assume that a manual selection of loops would also result in the shortest set of loops. We strongly doubt, however, that a manual and intuitive selection would be able to choose the smallest comprehensive set of feedback loops in larger models. This is because humans fail to account for the high degree of complexity embodied in a large feedback loop model. Thus, we advocate the use of Oliva's method to obtain the objective SILS of a mental model - a unique property of such a model.

After we have obtained the SILSs for both models, the entire set of $k$ feedback loops in models $A$ and $B$ must now be analyzed: we have to select the loops of both models which correspond to each other. This requires a semantic matching of the feedback loops. This semantic assessment accounts for the content of the loops which cannot be captured by a formal method. It considers the content which the loop captures, i.e., the causal mechanism it represents. The human coder decides if two loops correspond to one another, which makes the matching depend on the competences and knowledge of the coder. Unless a formalized procedure for this process is developed, it remains a step where careful and explicit reasoning is required. However, in most cases this assessment is straightforward for an experienced coder, as the application in Section 4 will show. The result of the semantic matching is the set of paired feedback loops, $L_{C}$; or the set of loops $A$ and $B$ have in common. In cases where no correspondence of a loop of $A$ with a loop of $B$ can be established, this first loop is marked as unique and the maximum loop distance is assigned. As a result, the loop sets $L_{u A}$, of the loops unique to $A$, and $L_{u B}$, the loops unique to $B$, are created.

The correspondences of the feedback loops can be represented as a quadratic matrix $L$ with $k \times k$ elements, using indexes $m$ and $n$ to refer to rows and columns, respectively. For instance, if there is a correspondence between two loops, then $l_{m n}=[1]$; otherwise $l_{m n}=[0]$. 
From $L$, we derive the loop distance ratio, $L D R(m, n)$. It indicates the degree of difference between a pair of loops. It is a number ranging from [0] ("identical") to [1] ("completely different"). For loops that are only elements of $L_{u A}$; or $L_{u B}$, where $l_{m n}=[0]$, and $L D R(m, n)=[1]$. In the other cases, the loop distance ratio is calculated using the element distance ratio of two corresponding loops, $E D R(m, n)$, the difference in polarities of these loops, $\operatorname{lpold}(m, n)$, and their difference in delay, $\operatorname{ld} d(m, n)$ (Eq. (3)). The calculation of $E D R(m, n)$ is demonstrated in Eq. (2). The feedback loops $m$ and $n$ have the same polarity, if $\operatorname{lpold}(m, n)=[0] ;[1]$ indicates a different polarity. The term $l d d(m, n)=[0]$ if both loops are equal in delays and [1] otherwise. The weights of the different terms are given by $\eta, l, \kappa ;$ with $\eta+l+\kappa=[1]$.

Equation 3: The loop distance ratio, $L D R$ calculates the distance ratio between respective loops of $A$ and $B$.

$$
L D R(m, n)=\eta^{*} \operatorname{ldd}(m, n)+l^{*} \operatorname{lpold}(m, n)+\kappa^{*} \operatorname{EDR}(m, n) .
$$

\subsection{The level of the complete loop set: The model distance ratio}

The distance of the set of all feedback loops is defined by the model distance ratio, $M D R(A, B)$. From a feedback loop perspective, two models are identical if they have a $\operatorname{MDR}(A, B)=[0]$; the models are perceived to be 'similar', if $[0]<M D R(A, B)<[0.25]$; both models are dissimilar if $\operatorname{MDR}(A, B)>[0.25]$. The model distance ratio is the plain average of all loop distance ratios (Eq. (4)).

Equation 4: The model distance ratio, $M D R$, is the plain average of the loop distance ratios of all loops of $A$ and $B$.

$$
\operatorname{MDR}(A, B)=\frac{\sum_{l=1}^{n} L D R(m, n)_{l}}{n}
$$

\section{Application}

In this section, we apply the method to compare two already explicated mental models, $A$ and $B$, which are represented as causal loop diagrams. This is an accepted form of representing participants' mental models (most recently, Doyle et al., 2008; Fokkinga et al., 2009). As discussed in the previous sections, our method accounts for all elements of MMDS that can be represented in causal loop diagrams (Lane, 2008; Richardson and Pugh, 1981; Sterman, 2000). We use the market growth model (Forrester, 1968a; Morecroft, 2007) - model $A$ - and a model of the growth and underinvestment archetype (Senge, 1990) - model B (Fig. 2, both models are taken from Morecroft, 2007: 194 and 198). These models fit our purpose since their structure is relatively simple and both are widely known and used in educational settings. In addition, each of them offers a different perspective on the dynamic situation of transition from accelerating growth towards crisis and decline. Using the both models, we can focus on the comparison and demonstrate our method. A major assumption is that the selected representations are professionally elicited mental models of relevant subjects. ${ }^{3}$

The original distance ratio (Markóczy and Goldberg, 1995), that is, the degree of similarity of both models without the consideration of delays and their feedback loops, is $D R(A, B)=58 \%$ (Schaffernicht and Groesser, 2009). This suggests that $A$ and $B$ are significantly different from one another. The application of our approach, which explicitly accounts for delays, feedback loops, and the polarities of feedback loops, yields a higher degree of similarity $(\operatorname{EDR}(A, B)=43 \%)$. Even though $A$ and $B$ still differ vastly in their content at the level of the elements, the difference has been reduced significantly by using our comparison method. The models are actually more similar when accounting for further details of dynamic systems. How do things go, then, when we analyze similarity on the level of feedback loops? The loop set of $A$ contains four loops; $B$ contains five loops. Each loop is assigned a unique identification number. The information concerning each loop's polarity, the number of delays and the number of links will be used to determine the distance between loops. Table 2 summarizes all the loops and their characteristics.

In the example, the loop sets of $A$ and $B$ are SILSs that we have confirmed using Oliva's method (2004). For establishing correspondences between the loops in both models, the semantical analysis has been used. Both loop \#1 and loop \#5 explain the growth in sales of an organization. The variables of both feedback loops which explain these mechanisms match also on the semantic level. The difference is that loop \#5 represents the growth mechanism more in detail. We conclude that loop \#1 and loop \#5 are identical on a semantic level. The correspondence between the other loops of $A$ and $B$ is established in the same way. Loop \#9 is unique and therefore cannot be reasonably matched to any loop of $A$. Consequently, the cells $\left[l_{1,5}, l_{2,6}, l_{3,7}, l_{4,8}\right]$ of the loop matrix $L$ have a value of [1]. In other words, four loops of $A$ and $B$ are identical $\left(L_{C}=[1=5,2=6,3=7,4=8] ; L_{u A}=[\emptyset], L_{u B}=[9]\right)$. These four pairs of loops must be used to calculate the loop distance ratios, $L D R s$, using Eq. (3). The results are provided in Table 3.

We note that each of the loop distance ratios (column "LDR") is significantly smaller than the distance ratio of Markóczy and Goldberg (1995) and the respective element distance ratios (column "EDR"). This puts forward the consideration that at the level of feedback loops $A$ and $B$ are less different than the element distance ratio suggests. By using Eq. (4), one ascertains that the distance between both models is $\operatorname{MDR}(A, B)=32 \%$. Note that without loop \#9, the distance between the models would be reduced to $14 \%$. In addition, an analysis of the sensitivity of the weights $(\eta, l$, and $\kappa)$ reveals that the $L D R s$ and the MDR become more similar to the EDR when the relative significance of the weight for the element distance ratio, $\kappa$, is increased. In this case, the differences in the elements between $A$ and $B$ gain more relevance. This consequence is straightforward, but as long as $\kappa$ does not exceed a threshold of 0.5 , the LDRs change only insignificantly. Besides these calculations, additional insightful statements about $A$ and $B$ are possible utilizing the sets which have already been introduced: $V_{C}, V_{u A}$, and $V_{u B}$ (level of elements) and $L_{C}, L_{u A}$, and $L_{u B}$ (level of loops). We use these sets to calculate the fractions between $A$ and $B$ at the respective levels (Table 4).

As Table 4 shows, $B$ is larger in terms of variables, links, and feedback loops than $A$. From model $A$ to $B$, 4 variables and 8 links have disappeared, while 7 variables, 14 links and one loop have been added: in each row of the table, more has been added than deleted. For a within-subject study, this difference may indicate that the subject's MMDS now represents the situation in a more detailed way. For a between-subjects study, this difference may signify that the second subject has a more detailed understanding of the situation. In both

\footnotetext{
${ }^{3}$ The issue of model elicitation is addressed in the discussion section. The paper's contribution concentrates on the method for comparing mental models, not on their elicitation. There is a range of methods for eliciting mental models all of which produce information that is well represented by causal loop diagrams.
} 


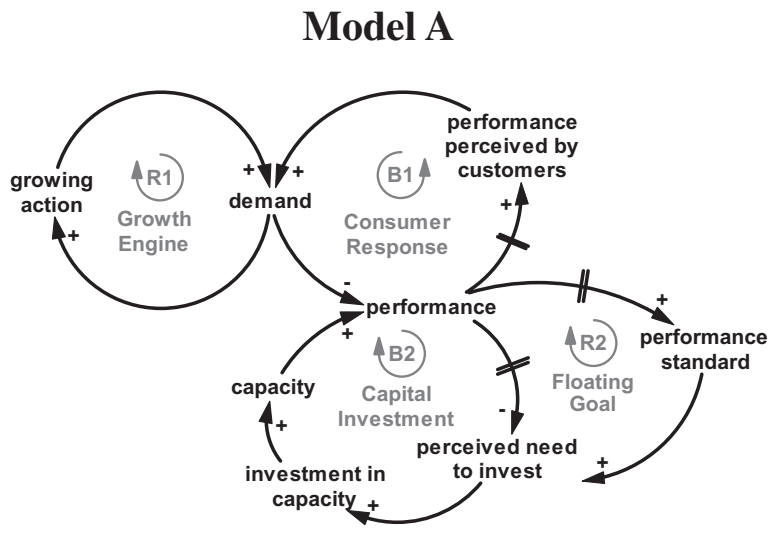

Model B

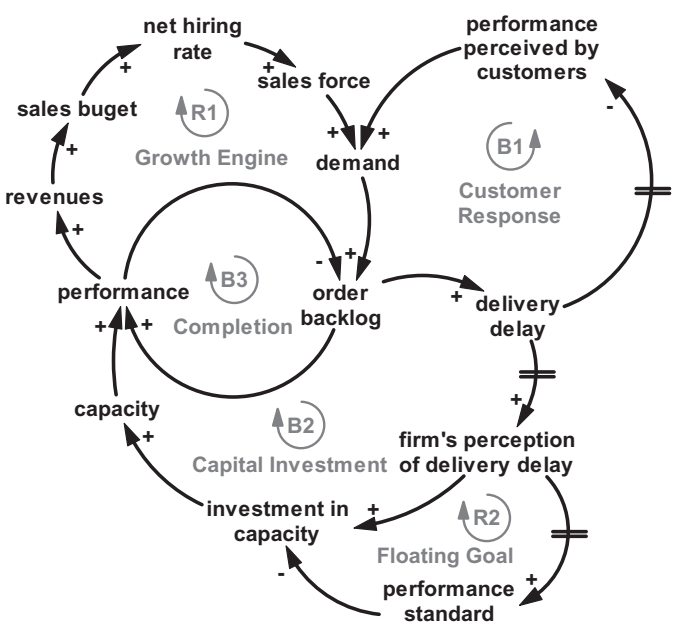

Fig. 2. Model of the growth and underinvestment archetype (Model A) and market growth model (Model B).

Table 2

List of the feedback loops in $A$ and $B$.

\begin{tabular}{llllll}
\hline Model & Loop & $\#$ & Polarity & \# of Delays & \# of Links \\
\hline A & R1 & 1 & + & 0 & 2 \\
A & B1 & 2 & - & 1 & 1 \\
A & R2 & 3 & + & 1 & 5 \\
A & B2 & 4 & - & 0 & 4 \\
B & R1 & 5 & - & 1 & 7 \\
B & B1 & 6 & + & 2 & 4 \\
B & R2 & 7 & - & 1 & 7 \\
B & B2 & 8 & - & 0 & 2 \\
B & B3 & 9 & & \\
\hline
\end{tabular}

Table 3

Loop distance ratios, $L D R s$, between $A$ and $B$.

\begin{tabular}{lllll}
\hline LDR & ldd & lpold & EDR & LDR \\
& $\eta$ & $l$ & $\kappa$ & \\
& 0,33 & 0,33 & 0,33 & $16 \%$ \\
\hline $1=5$ & 0 & 0 & $49 \%$ & $13 \%$ \\
$2=6$ & 0 & 0 & $40 \%$ & $13 \%$ \\
$3=7$ & 0 & 0 & $39 \%$ & $15 \%$ \\
$4=8$ & 0 & 0 & $44 \%$ & $100 \%$ \\
9 & n/a & n/a & n/a & $10 \%$ \\
\hline
\end{tabular}

Table 4

Additional indicators for comparing $A$ and $B$.

\begin{tabular}{|c|c|c|c|c|c|}
\hline \multirow[t]{2}{*}{ Item } & \multicolumn{3}{|c|}{ Unique to } & \multicolumn{2}{|l|}{ Common } \\
\hline & A & B & Growth & identical & different \\
\hline Variables & 4 & 7 & 0.50 & 5 & $(\mathrm{n} / \mathrm{a})$ \\
\hline Causal links & 8 & 14 & 0.55 & 3 & 0 \\
\hline Loops & 0 & 1 & 0.25 & 0 & 4 \\
\hline
\end{tabular}

cases, it is important to note that the differences are significant at the level of variables and links, but are relatively small at the level of loops. Thus, $A$ and $B$ differ only marginally in their dynamic properties; they show significant differences on the micro-level, but not on the macro-level.

An additional example demonstrates the importance of the level of feedback loops. Let us take a model $C$, which has only one loop (\#5) in common with $B$, but otherwise identical characteristics in terms of variables and links. The element distance ratio $E D R(C, B)=43 \%$ would be the same as in the previous example. However, the LDRs of the loops \#6, \#7, and \#8 would attain the maximal distance of 100\%. Consequently, the $\operatorname{MDR}(C, B)$ would significantly increase to $83 \%$, which is almost a threefold increase compared to $M D R(A, B)=31 \%$. In this example, no difference between $C$ and $B$ exists at the level of variables and links. On the contrary, the growth at the level of loops would 
be $400 \%$. In a within-subject study, this would suggest that the subject has hugely increased his dynamic understanding of a system. In a between- subjects study, it would mean that the subject in $C$ has a static understanding of the system, while $B$ 's understanding is highly dynamic. This example demonstrates that approaches that do not account for the level of feedback loops can be deceptive: two MMDSs may seem to be identical when analyzed only at the level of variables and links, but still be rather different at the level of feedback loops. This can only be detected when model ratios are calculated for each of the descriptive levels.

\section{Discussion}

In this section we discuss the contribution of the comparison method, reflect on the methodological characteristics and the method's relation to the issue of elicitation and measurement of mental models. As a final point, we elaborate on the method's limitations.

\subsection{Contribution}

The paper enhances existing approaches to compare MMDS in a twofold way: first, it broadens the scope of analysis, and second, it deepens the level of granularity. With respect to the first finding, existing approaches (e.g., Markóczy and Goldberg, 1995; Schvaneveldt, 1990) compare only variables, links, and the links' polarities. It has been shown that these approaches do not account for delays in causal effects, feedback loops, and loop polarities, all of which are fundamental conceptual elements of dynamic systems. This is a major step toward obtaining and comparing mental models that are more complex and therefore capable of representing reality more accurately. Mental model researchers have recently tried to incorporate some of these conceptual elements in their research (Doyle et al., 2008; Fokkinga et al., 2009; Gary, 2008; Gary and Wood, forthcoming). The method presented here advances this research stream by applying recent theoretical elaborations.

For the latter, our method provides also a more finely grained level of analysis of the MMDS. The existing comparison methods resulted in highly aggregated ratios whose interpretation does not allow for concrete assertions about the source of differences (Markoczy, 1997; Markóczy and Goldberg, 1995). In metaphorical terms, these ratios are but the tip of the iceberg, leaving the underlying structure unanalyzed. The paper's method addresses this issue by providing analyses at the level of variables and links, the level of feedback loops, and the level of the complete model. The method uses three descriptive ratios (EDR, LDR, MDR; Section 3) to indicate the level of difference of two MMDS at each level. As shown in the previous section, the inspection of these levels allows researchers to interpret the meaning and importance of the differences detected. The level of variables and links is closest to the results of existing approaches (Capelo and Dias, 2009; Markóczy and Goldberg, 1995; Schvaneveldt, 1990). The level of feedback loops now enables the researcher to compare relative levels of dynamic complexity ingrained in the mental model (Doyle et al., 2008; Senge, 1990). The loop distance ratio $L D R$ and the model distance ratio MDR support the attempts of current research to account for the dynamic characteristic (Fokkinga et al., 2009).

\subsection{Characteristics of the method}

Robustness: The results of a comparison between two models should be insensitive to changes in the comparison algorithm. Our method is robust against two main sources of such sensitivity. The first stems from the selection of the feedback loops that are to be compared; the second derives from the selection of weights for the individual parts of the distance ratios used for the computation.

For the first, even in main system dynamics literature (Morecroft, 2007; Richardson and Pugh, 1981; Sterman, 2000) there is no heuristic for selecting the feedback loops that shall be described or analyzed. With increasing model size, the number of possible feedback loops increases exponentially (Kampmann, 1996) and the recognition of feedback loops becomes puzzling. To minimize these challenges, we use the graph-theoretical model analysis of Oliva (2004) to cope with this complexity problem. The smallest independent loop set (SILS) ensures that an objective loop set is found that contains the smallest number of feedback loops that contain all endogenous variables. By means of this model analysis, we have been able to eliminate the complexity problem. Others have tried to control for the degree of feedback loop complexity (Doyle et al., 2008). The approach used here is superior since it avoids double counting of feedback loops and arrives at an objective set of feedback loop which is unique for each model.

The second issue was the selection of weights for the individual parts that are used for the calculation of the different distance ratios. For the LDRs, we have selected equal weights. A sensitivity analysis which changes the weights for the three components of the $L D R$-equation (Eq. (3)) by 50\% shows that the resulting LDRs vary only insignificantly with respect to the input changes. We therefore conclude that the selection of weights exerts only minor influences on the different ratios, as long as the element level is not weighted more than $50 \%$.

Qualitative analysis: Our method relies upon a qualitative matching of the individual feedback loops in both models. This qualitative analysis strongly influences the results on the level of feedback loops as well as on the complete model level. We must rely on this type of analysis since it concerns the semantics of the content, not the model's objective structure. The inter-subjectivity of the qualitative analysis can be improved by additional means, e.g., when taking into account the neighbouring and connected variables to interpret the variable's meaning (Eden, 1988; Eden and Ackermann, 1998). Moreover, a strict definition of the variables (for simulation models described by Coleman, 1964; Jacobsen and Bronson, 1987) reduces the conceptual ambiguity. The issue can be further mitigated by a strict elicitation and measuring approach, which we will address in the next section.

\subsection{Measurement}

In this paper, we assume that the mental models that are compared are elicited using a reliable, scientific method. This topic is not trivial, however. This is because existing methods for eliciting mental models are not designed to measure them, but to improve them (Doyle et al., 2008). When one employs the current elicitation methods, e.g.,causal loop diagrams (Lane, 2008; Richardson and Pugh, 1981; Sterman, 2000), one changes and therefore severely biases the mental models whenever no pre-intervention data is collected against which the post-intervention data can be compared. Some studies have tried to gather pre-intervention data, but these have met with only limited success, since robust elicitation methods are not available yet (Rouwette and Vennix, 2006). Recently, researchers have proposed new mea- 
surement instruments: they elicit narratives about aspects of a dynamic phenomenon, which then are reconstructed by researchers using such methods as causal loop diagrams (Fokkinga et al., 2009) or causal chain analysis (Doyle et al., 2008).

A different approach is to present the participants with pairs of pre-defined variables. The subjects then have to assign causal relations and polarities to these bivariate tuples (Gary, 2008; Gary and Wood, forthcoming; Plate, 2010). Markóczy and Goldberg (1995) and also Schvaneveldt (1990) have used this method to elicit mental models, because it shortcuts the complicated elicitation and coding process. A different version of this approach was developed by Desthieux et al. (2010). Using an computer-facilitated elicitation process, the subjects have to specify a small number of variables which were then used to elicit causal relations and polarities. We argue that the use of predefined or small amounts of variables influence subjects significantly and therefore biases the elicitation to a large degree.

Additional methods for eliciting specific parts of a mental model are available, e.g., to elicit shapes of nonlinear relationships (Ford and Sterman, 1997) or elicitation in group interactions (Vennix et al., 1992; Vennix and Gubbels, 1992). What we wish to emphasize, however, is that the characteristics of dynamic systems that we include in our comparison method can also guide research about elicitation. Thus, new instruments for measuring mental models may also account for link delays, loops, and loop polarities.

\subsection{Limitations}

It should be noted that the comparison method is based solely on information about the causal structure - that is, no behavioural aspects are taken into account. The reason for this choice is that, according to the currently accepted definition, mental models are structural representations. This assumption might be challenged in future research (Schaffernicht, 2010). However, the deduction of system behaviour based on a structural mental model is a topic that is not yet well understood. Before this issue can be addressed, mental model research has to account for the difference between stock and flow variables. Our method does not take this factor into account. Still, research about stock and flow thinking has demonstrated that the human capacity to deduce system behaviour from the stock and flow structure is severely limited (Cronin et al., 2009; Sterman, 2008; Sweeney and Sterman, 2007). Despite these findings, the scrutiny of stocks and flows in human mental models has not yet been attempted. Thus, this differentiation seems to be a useful elaboration that is well worth pursuing.

For our method, we use an implicit equality assumption: all variables, links, and subsequently loops are equally relevant. At the level of feedback loops, of course, this is not a valid representation of reality: the strengths of feedback loops are unequal and even shift over time (Kampmann and Oliva, 2006, 2008, 2009). However, since we compare only the structure of the MMDS, we cannot assert anything about shifts in the loops' relative significances. Thus, our method does not account for different loop strengths.

At the element level, one must also question the assumption that all variables and links are equally important. For instance, a variable that connects two or more loops might be more important than other variables. Also, to account for the different strengths of variables, links, and loops, the method would demand an algorithm that also accounts for shifts in the relevance of structure over time.

\section{Conclusion}

Research about mental models of dynamic systems has gained attention in the operational research community. In particular, insights into the conceptual elements of mental models are important for understanding human decision making in dynamic environments. Extensive research has demonstrated that although humans are most often not capable of forming adequate mental models of dynamic systems, none the less the capability of dynamic thinking can be enhanced (Sterman, 2010).

Until now, the exploration of the content and structure of mental models has used a diversity of representational and comparison approaches, mainly either the distance ratio (Markóczy and Goldberg, 1995) or the closeness ratio (Schvaneveldt, 1990). As a consequence, the results from these different studies are difficult to compare, and both the accumulation of knowledge and the subsequent progress of research are limited. Furthermore, the existing approaches neglect relevant conceptual elements and levels of description of dynamic systems. They therefore oversimplify the complexities of such dynamic systems and lead to insufficient representations of them. To overcome these shortfalls, we enhance the distance ratio approach to account for relevant characteristics of dynamic systems. By considering delays in causal links, feedback loops, and the polarities of feedback loops, we make it possible to analyze and compare mental models more comprehensively, enabling the detection of differences between mental models at three levels: the element level (element distance ratio, EDR), the level of the individual feedback loop (loop distance ratio, $L D R$ ), and the level of the complete model (model distance ratio, MDR). An application of the method shows that this previously non-existent information concerning the feedback loops is essential for understanding differences between mental models of dynamic systems.

The comprehensive method proposed here can be used to compare several versions of mental models both within and between subjects. In the former case, the effects of exposing subjects to simulators, interactive learning environments, and other educational devices-but also modelling interventions-on MMDS can now be studied in a way that allows cross-study comparison and accumulation. Also, systems thinking repertory (Sweeney and Sterman, 2007) and other studies belonging to that group can generate more comparable results. In other words, we believe this method can help most operational researchers interested in mental models of dynamic systems as a means for shaping decisions and decision policies. Future research will allow one to incorporate the distinction between different types of variables into the comparison; also, behavioural inferences deduced from the causal structure may become accessible to elicitation and thus comparable.

\section{Acknowledgements}

We thank Martin Kunc, Markus Schwaninger, two anonymous referees of EJOR, participants of a research session at the International System Dynamics Conference 2009, Albuquerque, USA, and four anonymous reviewers of this conference, for their helpful comments.

\section{References}

Andersen, D.F., Richardson, G.P., Vennix, J.A.M., 1997. Group model building: Adding more science to the craft. System Dynamics Review 13 (2), $187-201$. Bakken, B.E., 1993. Learning and Transfer of Understanding in Dynamic Decision Environments. Unpublished Ph.D. Thesis, M.I.T. 


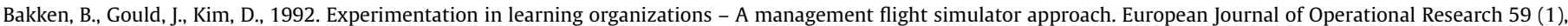
167-182.

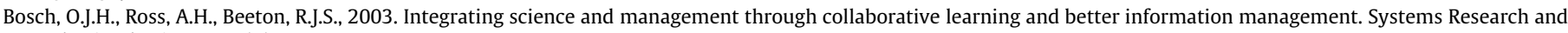
Behavioral Science 20 (2), 107-118.

Calori, R., Johnson, G., Sarnin, P., 1994. CEOs' cognitive maps and the scope of the organization. Strategic Management Journal 15 (6), $437-457$.

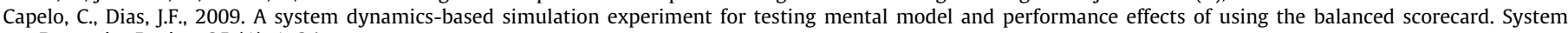
Dynamics Review 25 (1), 1-34.

Carley, K., Palmquist, M., 1992. Extracting, representing, and analyzing mental models. Social Forces 70 (3), 601-636.

Coleman, J.S., 1964. Introduction to Mathematical Sociology, second print. ed. Free Press, New York.

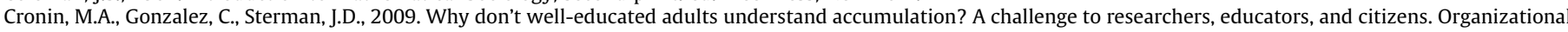
Behavior and Human Decision Processes 108 (1), 116-130.

Desthieux, G., Joerin, F., Lebreton, M., 2010. Ulysse: A qualitative tool for eliciting mental models of complex systems. System Dynamics Review 26 (2), $163-192$.

Doyle, J.K., Ford, D.N., 1998. Mental models concepts for system dynamics research. System Dynamics Review 14 (1), 3-29.

Doyle, J.K., Ford, D.N., 1999. Mental models concepts revisited: Some clarifications and a reply to lane. System Dynamics Review 15 (4), 411-415.

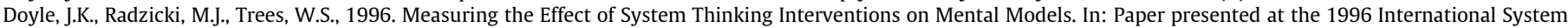
Dynamics Conference, Cambridge, Massachusetts.

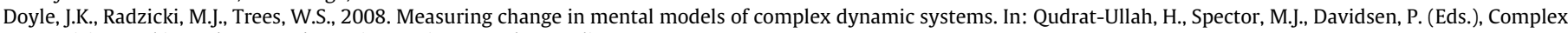
Decision Making: Theory and Practice. Springer-Verlag, Berlin, pp. 269-294.

Eden, C., 1988. Cognitive mapping. European Journal of Operational Research 36 (1), 1-13.

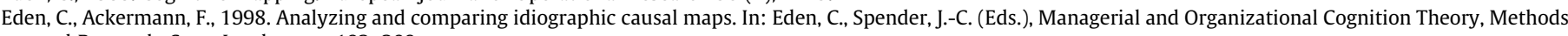
and Research. Sage, London, pp. 192-209.

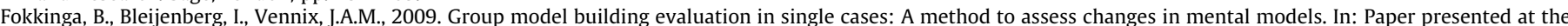
27th International Conference of the System Dynamics Society, New Mexico, USA.

Ford, D.N., Sterman, J.D., 1997. Expert knowledge elicitation to improve formal and mental models. System Dynamics Review 14 (3), $309-340$.

Forrester, J.W., 1961. Industrial Dynamics. Productivity Press, Cambridge, MA.

Forrester, J.W., 1968a. Market growth as influenced by capital investment. Industrial Management Review 9 (2), 83-105.

Forrester, J.W., 1968b. Principles of Systems. The MIT Press, Cambridge.

Forrester, J.W., 1992. Policies, decisions and information-sources for modeling. European Journal of Operational Research 59 (1), $42-63$.

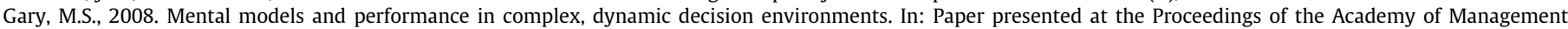
Conference, Anaheim, USA.

Gary, M.S., Wood, R.E., (forthcoming). Mental Models, Decision Rules, and Performance Heterogeneity. Strategic Management Journal.

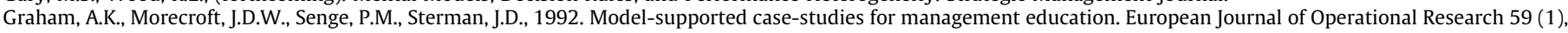
151-166.

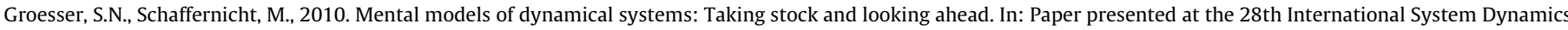
Conference, Seoul, South-Korea.

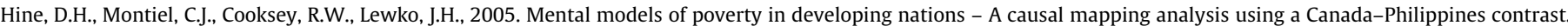
Journal of Cross-Cultural Psychology 36 (3), 283-303.

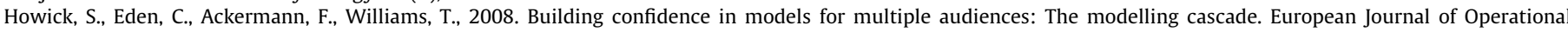
Research 186 (3), 1068-1083.

Jacobsen, C., Bronson, R., 1987. Defining sociological concepts as variables for system dynamics modeling. System Dynamics Review 3 (1), 1-7.

Johnson-Laird, P.N., 1999. Deductive reasoning. Annual Review of Psychology 50, 109-135.

Johnson-Laird, P.N., 2001. Mental models and deduction. Trends in Cognitive Sciences 4 (10), 434-442.

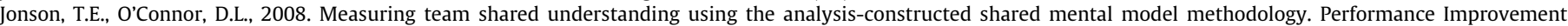
Quarterly 21 (3), 113-135.

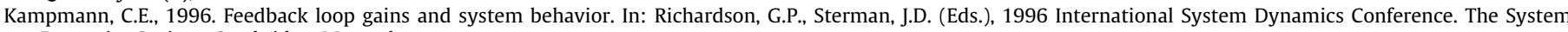
Dynamics Society, Cambridge, Massachusetts.

Kampmann, C.E., Oliva, R., 2006. Loop eigenvalue elasticity analysis: Three case studies. System Dynamics Review 22 (2), 141-162.

Kampmann, C.E., Oliva, R., 2008. Structural dominance analysis and theory building in system dynamics. Systems Research and Behavioral Science 25 (4), 505-519.

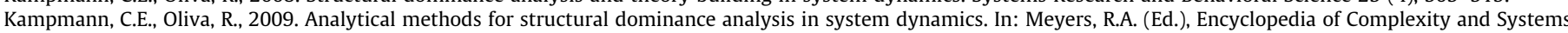
Science. Springer, New York, pp. 8948-8967.

Kluge, A., 2008. Performance assessments with microworlds and their difficulty. Applied Psychological Measurement 32 (2), 156-180.

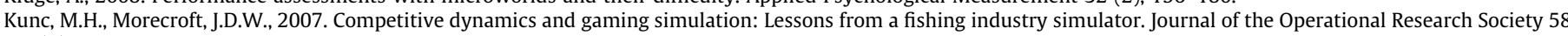
(8), 1146-1155.

Lane, D.C., 2008. The emergence and use of diagramming in system dynamics: A critical account. Systems Research and Behavioral Science 25 (1), 3-23.

Langan-Fox, J., Code, S., Langfield-Smith, K., 2000. Team mental models: Techniques, methods, and analytic approaches. Human Factors 42 (2), $242-271$.

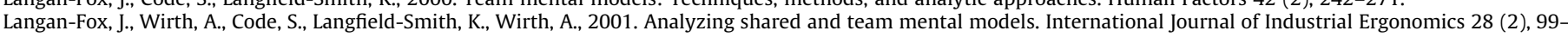
112.

Langfield-Smith, K., Wirth, A., 1992. Measuring differences between cognitive maps. Journal of the Operational Research Society 43 (12), $1135-1150$.

Langley, P.A., Morecroft, J.D.W., 2004. Performance and learning in a simulation of oil industry dynamics. European Journal of Operational Research 155 (3), $715-732$.

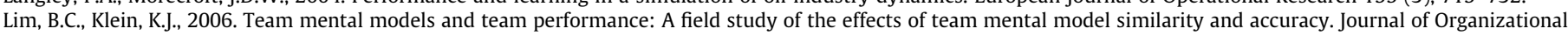
Behavior 27 (4), 403-418.

Maani, K.E., Cavana, R.Y., 2007. Systems Thinking, softcover ed.. System Dynamics: Managing Change and Complexity Pearson Education, New Zealand.

Markoczy, L., 1997. Measuring beliefs: Accept no substitutes. Academy of Management Journal 40 (5), $1228-1242$.

Markoczy, L., 2000. National culture and strategic change in belief formation. Journal of International Business Studies 31 (3), $427-442$.

Markóczy, L., Goldberg, J., 1995. A method for eliciting and comparing causal maps. Journal of Management 21 (2), 305-333.

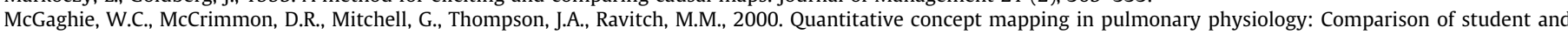
faculty knowledge structures. Advances in Physiology Education 23 (1), 72-81.

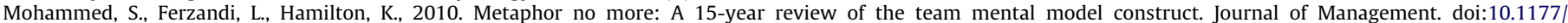
0149206309356804

Morecroft, J.D.W., 1992. Executive knowledge, models and learning. European Journal of Operational Research 59 (1), 9-27.

Morecroft, J.D.W., 2007. Strategic modelling and business dynamics: A feedback systems approach. John Wiley \& Sons, Chichester.

Moxnes, E., 1998. Not only the tragedy of the commons: Misperceptions of bioeconomics. Management Science 44 (9), 1234-1248.

Moxnes, E., 2004. Misperceptions of basic dynamics: The case of renewable resource management. System Dynamics Review 20 (2), $139-162$.

Oliva, R., 2004. Model structure analysis through graph theory: Partition heuristics and feedback structure decomposition. System Dynamics Review 20 (4), $313-336$.

Plate, R., 2010. Assessing individuals' understanding of nonlinear causal structures in complex systems. System Dynamics Review 26 (1), 19-33.

Richardson, G.P., 1999. Feedback thought in social science and systems theory. Pegasus Communications, Waltham, MA.

Richardson, G.P., Andersen, D.F., 1995. Teamwork in group model building. System Dynamics Review 11 (2), $113-137$.

Richardson, G.P., Pugh III, A.L., 1981. Introduction to System Dynamics Modeling with DYNAMO. Productivity Press, Cambridge, MA.

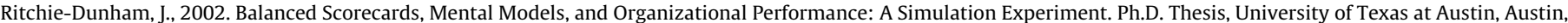
TX.

Romme, A.G.L., 2004. Perceptions of the value of microworld simulation: Research note. Simulation and Gaming 35 (3), 427-436.

Rouwette, E.A.J.A., Vennix, J.A.M., 2006. System dynamics and organizational interventions. Systems Research and Behavioral Science 23 (4), 451-466.

Rouwette, E.A.J.A., Vennix, J.A.M., van Mullekom, T., 2002. Group model building effectiveness: A review of assessment studies. System Dynamics Review 18 (1), $5-45$.

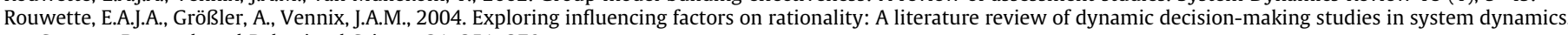
Systems Research and Behavioral Science 21, 351-370.

Rowe, A., Cooke, N., 1995. Measuring mental models: Choosing the right tools for the job. Human Resource Development Quarterly 6 (3), $243-255$. 


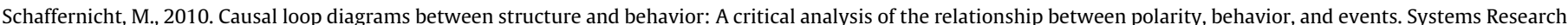
and Behavioral Science, published online: April 26, 2010.

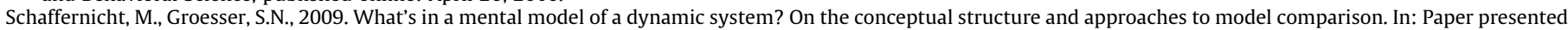
at the 27th International Conference of the System Dynamics Society, New Mexico, USA.

Schvaneveldt, R., 1990. Pathfinder associative networks. In: Schvaneveldt, R. (Ed.), Studies in Knowledge Organization. Ablex, Norwood, NJ.

Senge, P.M., 1990. The Fifth Discipline: The Art and Practice of the Learning Organization. Currency \& Doubleday, New York.

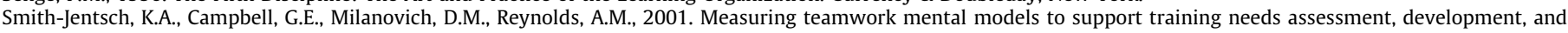
evaluation: Two empirical studies. Journal of Organizational Behavior 22, 179-194.

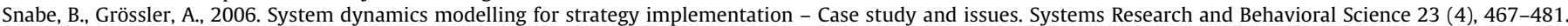

Sterman, J.D., 1985. A behavioral-model of the economic long-wave. Journal of Economic Behavior and Organization 6 (1), 17-53.

Sterman, J.D., 2000. Business Dynamics: Systems Thinking and Modeling for a Complex World. McGraw-Hill, Boston.

Sterman, J.D., 2008. Risk communication on climate: Mental models and mass balance. Science 322 (24 October), $532-533$.

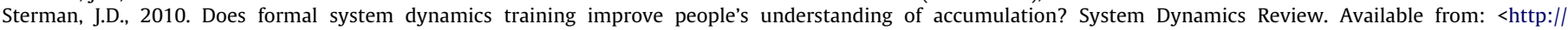
onlinelibrary.wiley.com/doi/10.1002/sdr.447/abstract>.

Sweeney, L.B., Sterman, J.D., 2007. Thinking about systems: Student and teacher conceptions of natural and social systems. System Dynamics Review 23 (2-3), $285-312$.

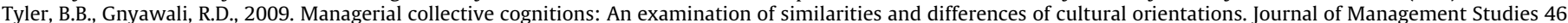
(1), 93-126.

Vennix, J.A.M., 1996. Group Model Building: Facilitating Team Learning Using System Dynamics. Wiley, Chichester.

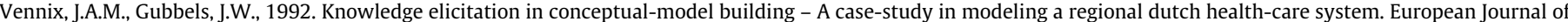
Operational Research 59 (1), 85-101.

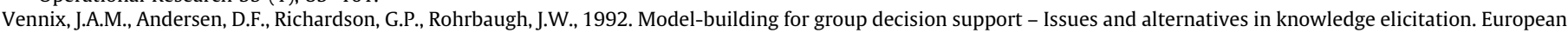
Journal of Operations Research 59 (1), 28-41.

Verburgh, L., 1994. Participative Policy Modeling Applied to the Health Care Insurance Industry. Doctorial Dissertation, University of Nijmegen, Nijmegen.

Weber, M., Schwaninger, M., 2002. Transforming an agricultural trade organization: A system-dynamics-based intervention. System Dynamics Review 18 (3), $381-401$.

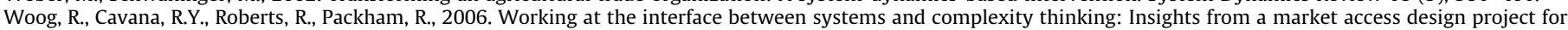
poor livestock producers. Systems Research and Behavioral Science 23 (6), 727-741. 